\title{
Frizzled-2: A potential novel target for molecular pancreatic cancer therapy
}

\author{
MINORU TOMIZAWA ${ }^{1}$, FUMINOBU SHINOZAKI ${ }^{2}$, TAKAO SUGIYAMA ${ }^{3}$, \\ SHIGENORI YAMAMOTO ${ }^{4}$, MAKOTO SUEISHI $^{3}$ and TAKANOBU YOSHIDA ${ }^{5}$
}

\author{
Departments of ${ }^{1}$ Gastroenterology, ${ }^{2}$ Radiology, ${ }^{3}$ Rheumatology, ${ }^{4}$ Pediatrics and ${ }^{5}$ Internal Medicine, \\ National Hospital Organization, Shimoshizu Hospital, Yotsukaido, Chiba 284-0003, Japan
}

Received May 4, 2013; Accepted November 7, 2013

DOI: $10.3892 / \mathrm{ol} .2013 .1681$

\begin{abstract}
In the present study, pancreatic cancer cell proliferation was analyzed following the suppression of frizzled (Fz)2 expression. Reverse transcription polymerase chain reaction (PCR) was performed using RNA isolated from pancreatic cancer cell lines, PANC-1, NOR-P1, PK-45H, PK-1, PK-59, MIA-Paca2 and KP4. A surgical specimen of pancreatic cancer was immunostained with antibodies specific to Fz2. Cell proliferation assays were performed with MIA-Paca2 cells transfected with small interfering RNA (siRNA) or short hairpin RNA (shRNA) of Fz2. Fz2 was found to be expressed in all pancreatic cancer cell lines, with the exception of NOR-P1. Immunostaining revealed that Fz2 was not expressed in normal pancreatic tissues, while it was expressed in pancreatic cancer cells. The expression levels of cyclin D1 were analyzed by quantitative PCR. The proliferation and expression of cyclin D1 were suppressed with the siRNA and shRNA of Fz2 in the MIA-Paca2 cells. Therefore, Fz2 is a potential target for the molecular therapy of pancreatic cancer.
\end{abstract}

\section{Introduction}

Pancreatic cancer is the fourth leading cause of cancer-related mortality (1). Furthermore, pancreatic cancer has a poor prognosis, with a 5-year survival rate of $6 \%$ (1), in part due to its ability to metastasize at an early stage (2). Non-cohesive pancreatic cancer is caused by the loss of expression of certain proteins, including E-cadherin, which results in cancer cell dissemination and subsequently, a poor prognosis $(3,4)$. Consequently, only $10 \%$ of patients with pancreatic cancer are treated with curative therapy, whilst $90 \%$ are treated conservatively (5). Therefore, there is a requirement for the development of novel treatments for pancreatic cancer.

Correspondence to: Dr Minoru Tomizawa, Department of Gastroenterology, National Hospital Organization, Shimoshizu Hospital, 934-5 Shikawatashi, Yotsukaido, Chiba 284-0003, Japan E-mail: nihminor-cib@umin.ac.jp

Key words: frizzled genes, $\beta$-catenin, quantitative PCR, MTS assay
Small-molecule receptor tyrosine kinase inhibitors are currently under investigation as anticancer agents (6). Insulin-like growth factor-I receptor inhibitors are particularly promising, as they inhibit the dissemination of pancreatic cancer cells $(7,8)$. One of the disadvantages of these inhibitors, however, is the observed co-inhibition of the insulin receptor (9).

The Wnt signaling pathway is pivotal for cell growth and differentiation. There are 3 signaling branches of the Wnt pathway, the canonical, planar-cell polarity and Wnt-Ca ${ }^{2+}$ pathways (10), with the former being the best characterized. During the canonical Wnt pathway, $\beta$-catenin is first phosphorylated and degraded in normal cells, following which, it enters the nucleus where it increases the expression of target genes, including cyclin D1, a protein involved in cell proliferation (11). Frizzled (Fz) is a receptor of Wnt ligand. Upon binding of Wnt ligand to Fz, the Wnt pathway is activated. In total, 10 members of the Fz family of genes have been identified based on structural and functional homology studies (12). Fz genes have been previously implicated in carcinogenesis and embryogenesis and their expression is upregulated in gastric cancer and hepatoma cells $(13,14)$. We investigated the possibility of Fz2 as a potential target of molecular therapy for pancreatic cancer.

\section{Materials and methods}

Cell culture. Pancreatic cancer cell lines, PANC-1, MIA-Paca2, NOR-P1, PK-45H, PK-1, PK-59 and KP4, were purchased from Cell Bank, RIKEN BioResource Center (Tsukuba, Japan). MIA-Paca2 was cultured in Dulbecco's modified Eagle's medium (DMEM), KP4 was cultured in DMEM:F12 and the remaining lines were cultured in Roswell Park Memorial Institute medium-1640. Media were purchased from Sigma-Aldrich (St. Louis, MO, USA) and supplemented with $10 \%$ fetal bovine serum (FBS; Life Technologies, Carlsbad, CA, USA). The cell lines were cultured with $5 \%$ carbon dioxide at $37^{\circ} \mathrm{C}$ in a humidified chamber.

Reverse transcription (RT) and quantitative polymerase chain reaction $(P C R)$. The cells were spread in 6-well plates (Asahi Glass Co., Ltd., Tokyo, Japan) and cultured. When the cells 
had reached $80 \%$ confluency, they were further cultured for $48 \mathrm{~h}$ after transfection. Total RNA (5 $\mu \mathrm{g})$, isolated with Isogen (Nippon Gene Co., Ltd., Tokyo, Japan), was used to generate cDNA with Super Script III and oligo dT primers, according to the manufacturer's instructions (Life Technologies). Human whole pancreas RNA was purchased from Takara and used as a positive control (Takara Bio, Inc., Shiga, Japan). The PCR primers, annealing temperatures, reaction cycle numbers and product sizes were as follows: Fz1 (GenBank accession no. NM_003505) forward (F), 5'-AATGACAAGTTCGCC GAGGAC-3' and reverse (R), 5'-GCCAGGTGAAAATACTGT GAGTTGG-3' (59 ${ }^{\circ} \mathrm{C}$ for 30 cycles; 206 bp); Fz2 (NM_001466) F, 5'-CAAGGTGCCATCCTATCTCAGC-3' and R, 5'-GTA GCAGCCCGACAGAAAAATG-3' $\left(59^{\circ} \mathrm{C}\right.$ for 30 cycles; 247 bp); Fz3 (NM_017412) F, 5'-AGAGAAGAACTGTCATTT GCTCGC-3' and R, 5'-TCCTTGTGTCACTGTGGAAGCC-3' $\left(53^{\circ} \mathrm{C}\right.$ for 30 cycles; 255 bp); Fz4 (NM_012193) F, 5'-CAAGTG ATTCTCCTGCCACAGC-3' and R, 5'-CAACTCTCTCCA GTGTCCTCCATC-3' $\left(57^{\circ} \mathrm{C}\right.$ for 30 cycles; $\left.270 \mathrm{bp}\right)$; Fz5 (NM_003468) F, 5'-CCCTCATCCCCTAAGAGAGAC AAAG-3' and R, 5'-GCTGGCTGTGAAGAAGTTGCTG-3' (55 ${ }^{\circ} \mathrm{C}$ for 30 cycles; 230 bp); Fz6 (NM_003506) F, 5'-AGCAGC ATCCATCTCCAGACTCTC-3' and R, 5'-CTGAATGACAAC CACCTCCCTG-3', (57 ${ }^{\circ} \mathrm{C}$ for 30 cycles, $\left.251 \mathrm{bp}\right) ; \mathrm{Fz} 7$ (NM_003507) F, 5'-AGACTTAGCCACAGCAGCAAGG-3' and R, 5'-CGCCGTTATCATCATCTTCCTG-3' (58 ${ }^{\circ} \mathrm{C}$ for 30 cycles; 287 bp); Fz8 (NM_031866) F, 5'-ATCCAAAGCAGA TGCCATTGTC-3' and R, 5'-AACACTGTGAAGGGGTGG GAAC-3' (59 ${ }^{\circ} \mathrm{C}$ for 30 cycles; 137 bp); Fz9 (BC_026333) F, 5'-TCTTTGGAGAACCCCACACACC-3' and R, 5'-TGC TCACTTGCCTGACCTTGAC-3' (60 ${ }^{\circ} \mathrm{C}$ for 30 cycles; $\left.148 \mathrm{bp}\right)$; Fz10 (NM_007197) F, 5'-AAACGCTGGACTGCCTGATG-3' and $\mathrm{R}, 5^{\prime}$-GCTTTTTTGTAAATCCCACCGC-3' $\left(58^{\circ} \mathrm{C}\right.$ for 30 cycles; 217 bp); and GAPDH (NM_002046) F, 5'-ACCTGA CCTGCCGTCTAGAA-3' and R, 5'-TCCACCACCCTGTTG CTGTA-3' (63 $\mathrm{C}$ for 30 cycles; $246 \mathrm{bp}$ ). PCR was performed using Taq DNA polymerase (Life Technologies) and products were subjected to analysis by gel electrophoresis in $2 \%$ agarose in 1X TAE (40 mM Tris-acetate/1 mM EDTA). Quantitative PCR was performed using Fast SYBR Green Master Mix (Life Technologies) and analyzed with the MiniOpticon Detection System (Bio-Rad, Hercules, CA, USA). The primer pairs for quantitative PCR and the resultant product sizes were as follows: Fz2 (NM_001466) F, 5'-TCCTCAAGGTGCCAT CCTATCTC-3' and R, 5'-TGGTGACAGTGAAGAAGGTGG AAG-3' (183 bp); cyclin D1 (NM_053056) F, 5'-AGAGGCGGA GGAGAACAAACAG-3' and R, 5'-AGGCGGTAGTAGGAC AGGAAGTTG-3' (180 bp); and ribosomal protein L19 (RPL19; BC095445) F, 5'-CGAATGCCAGAGAAGGTCAC-3' and R, 5'-CCATGAGAATCCGCTTGTTT-3' (157 bp). Quantitative PCR was performed for 40 cycles of $5 \mathrm{sec}$ for denaturation and $5 \mathrm{sec}$ for annealing/extension. GAPDH and RPL19 were used as internal controls.

Immunostaining and microscopy. Serial sections of human pancreatic cancer tissue (62-year-old male with adenocarcinoma; BioChain Institute, Inc., Newark, CA, USA) were deparaffinized, autoclaved and incubated first with hydrogen peroxide, followed by a $30-\mathrm{min}$ incubation with $2 \%$ normal goat serum in phosphate-buffered saline (PBS; washing buffer). Following overnight incubation with a rabbit polyclonal anti-Fz2 antibody (1:5,000; Sigma-Aldrich), the specimens were rinsed with PBS and subsequently incubated with horseradish peroxidase-labeled anti-rabbit antibody (1:500; GE Healthcare, Amersham, UK) for 2 h. Next, diaminobenzidine (Dako, Carpinteria, CA, USA) was applied to the tissue sections as a chromogen, and the nuclei were stained with hematoxylin (Muto Pure Chemicals Co., Ltd., Tokyo, Japan) for $15 \mathrm{sec}$. The specimens were observed and images were captured under an AX80 microscope (Olympus Corp., Tokyo, Japan).

Cell proliferation analysis. The MIA-Paca2 cells were trypsinized, harvested and spread onto 96-well flat-bottom plates (Asahi Techno Glass) at a density of 1,000 cells per well. Next, the cells were incubated for $24 \mathrm{~h}$ in DMEM supplemented with $10 \%$ FBS. Following culture, the cells were transfected with the short interference RNA (siRNA) of Fz2 (siRNA-Fz2) or the short hairpin RNA (shRNA) of Fz2 (shRNA-Fz2) for $72 \mathrm{~h}$. Cell cultures were then used in 3-(4,5-dimethylthiazol-2-yl)-5-(3-carboxymethoxyphenyl)2-(4-sulfophenyl)-2H-tetrazolium inner salt (MTS) assays, according to the manufacturer's instructions (Promega Corporation, Madison, MI, USA). MTS is bio-reduced by cells into a colored formazan product that reduces absorbance at $490 \mathrm{~nm}$. Absorbance was analyzed at a wavelength of $490 \mathrm{~nm}$ with an iMark Microplate Absorbance Reader (Bio-Rad).

siRNA and shRNA transfection. Transfection of the cells with siRNA-Fz2 (Life Technologies) was carried out using Lipofectamine 2000 and Opti-MEM (both Life Technologies), according to the manufacturer's instructions. Briefly, siRNA and Lipofectamine 2000 were separately diluted in Opti-MEM at room temperature for $5 \mathrm{~min}$. The diluted siRNA and Lipofectamine 2000 were then incubated together for an additional $20 \mathrm{~min}$ at room temperature to facilitate complex formation. Next, culture medium was aspirated from dishes or wells containing cells and the complexes were added to cultured cells. shRNA-Fz2 (OriGene Technologies Inc., Rockville, MD, USA) was transfected into cells using Lipofectamine LTX (Life Technologies), according to the manufacturer's instructions. Briefly, shRNA was incubated with PLUS reagent for 5 min, following which, LTX reagent was added. A 30-min incubation at room temperature ensued and the complex was subsequently applied to the cell culture medium.

Statistical analysis. Cell proliferation and quantitative PCR data were analyzed by a one-way analysis of variance. The statistical analysis was performed using JMP 5.0 software (SAS Institute Inc., Cary, NC, USA) and $\mathrm{P}<0.05$ was considered to indicate a statistically significant difference.

\section{Results}

Fz gene expression levels in pancreatic tissue. The expression levels of the Fz genes were analyzed by RT-PCR in the normal pancreatic tissue and the pancreatic cancer cell lines (PANC-1, MIA-Paca2, NOR-P1, PK-45H, PK-1, PK-59 and KP4; Fig. 1A). Fz1, Fz3, Fz7 and Fz10 were expressed not only 

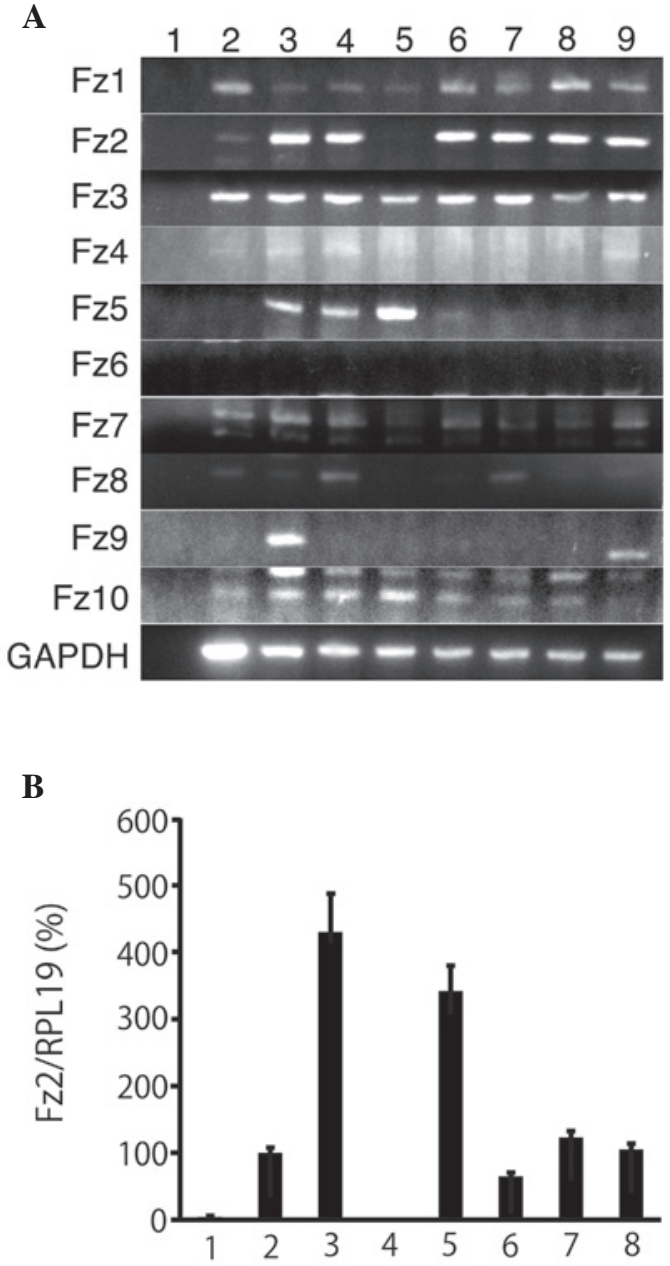

Figure 1.Expression of Fz genes in pancreatic cancer cell lines. (A) Expression of Fz genes was analyzed in pancreatic cancer cell lines by RT-PCR. Lane $1, \mathrm{H}_{2} \mathrm{O}$; 2, normal pancreatic tissue; 3, PANC-1; 4, MIA-Paca2; 5, NOR-P1; 6, PK-45H; 7, PK-59; 8, PK-1; and 9, KP4. Fz2/RLP19 was calculated as the expression level of Fz2. All experiments were performed in triplicate. (B) Expression levels of Fz2 were analyzed in pancreatic cancer cell lines by quantitative PCR Lane 1, normal pancreas; 2, MIA-Paca2; 3, PANC-1; 4, NOR-P1; 5, PK-45H; 6, PK-59; 7, PK-1; and 8, KP4. Fz, frizzled; RT-PCR, reverse transcription polymerase chain reation; RPL19, ribosomal protein L19.

in the pancreatic cancer cells, but also in the normal pancreatic tissues, while Fz6 was not expressed in either of the two cell types. Fz4 was only weakly expressed in the pancreatic cancer cell lines. In addition, Fz5 and Fz9 were not expressed in the normal pancreatic tissues, but were expressed in 3 and 1 of the pancreatic cancer cell lines, respectively. Fz8 was expressed only in the normal pancreatic tissues, while Fz2 was weakly expressed in the normal pancreatic tissues and expressed in all other pancreatic cancer cell lines examined, with the exception of NOR-P1. Relative to its expression in MIA-Paca2, the expression levels of Fz2 in the normal pancreatic tissue was $4.1 \pm 1.8 \%$ (Fig. 1B). The expression of Fz2 was $1.3 \pm 0.7 \%$ of NOR-P1. These expression results were consistent with the RT-PCR results, and Fz2 was consequently used for further analysis.

Immunostaining. Immunostaining was performed to determine the expression of Fz2 at the protein level in the normal pancreatic and pancreatic cancer tissues (Fig. 2).
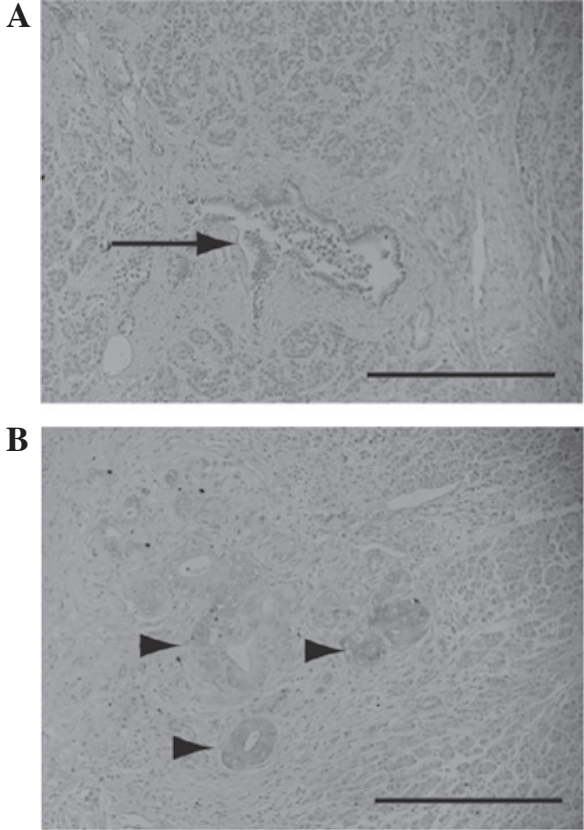

Figure 2. Immunostaining. Surgical specimens of (A) normal pancreatic duct tissues were compared with (B) specimens obtained from a patient with pancreatic cancer and immunostained with an antibody against Fz2. Arrow indicates the normal pancreatic duct and arrowheads indicate cancer (magnification, $\mathrm{x} 200$; scale bar, $100 \mu \mathrm{m})$. Fz2, frizzled-2.

Fz2 was not expressed in the glands or normal pancreatic duct tissues (Fig. 2A). By contrast, Fz2 was expressed in the pancreatic cancer cells (Fig. 2B).

Inhibition of $F z 2$ expression. These results prompted the study of the suppression of pancreatic cancer cell proliferation following the inhibition of Fz2 expression. MIA-Paca2 cells were cultured with siRNA- or shRNA-Fz2 and subjected to MTS assays. Relative to the mock transfection, the proliferation of the MIA-Paca2 cells was suppressed to $34.5 \pm 2.8 \%$ ( $\mathrm{P}<0.05$; Fig. 3A) following transfection with $200 \mathrm{nM}$ siRNA-Fz2, and to $68.0 \pm 19.0 \%(\mathrm{P}<0.05)$ following transfection with $100 \mathrm{ng} /$ well shRNA-Fz2 (Fig. 3B). The expression levels of Fz2 were analyzed by quantitative PCR to confirm suppression with siRNA- or shRNA-Fz2 (Fig. 4). Fz2 expression was suppressed to $12.9 \pm 0.9 \%(\mathrm{P}<0.05)$ following transfection with $200 \mathrm{nM}$ siRNA-Fz2 (Fig. 4A), and to $42.0 \pm 4.1 \%(\mathrm{P}<0.05)$ following transfection with $2.5 \mu \mathrm{g} / \mathrm{well}$ shRNA-Fz2 (Fig. 4C).

Cyclin D1 expression. Finally, the expression of cyclin D1 was analyzed to investigate the mechanism of the observed suppression of cell proliferation in the MIA-Paca2 cells. Fz2 expression levels were suppressed to $8.2 \pm 3.7 \%(\mathrm{P}<0.05)$ following transfection with $200 \mathrm{nM}$ siRNA-Fz2 (Fig. 4B), and to $26.2 \pm 2.6 \%(\mathrm{P}<0.05)$ following transfection with $2.5 \mu \mathrm{g} / \mathrm{well}$ shRNA-Fz2 (Fig. 4D).

\section{Discussion}

Sagara et al (1998) reported that Fz2 was not expressed in the normal pancreas (15). Although the RT-PCR results of the current study revealed a weak band of Fz2 expression in the normal pancreatic tissue, the expression was significantly low. 
A

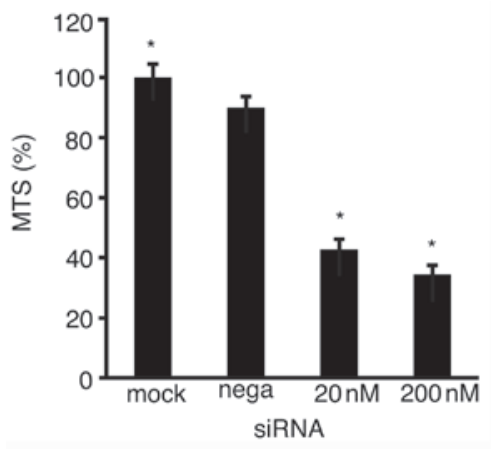

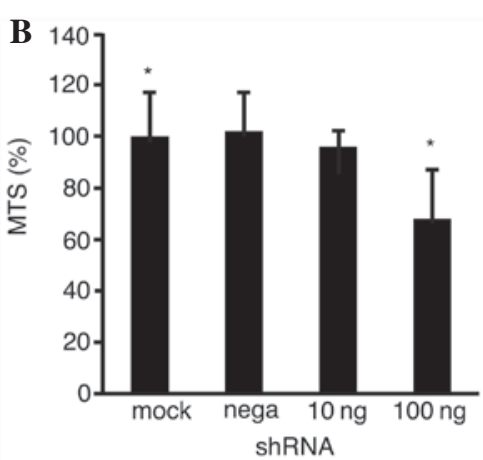

Figure 3. Cell proliferation analysis. Cell proliferation was analyzed in MIA-Paca2 cells by MTS assays $72 \mathrm{~h}$ after the transfection of cells with (A) the siRNA or (B) shRNA of Fz2. "P $<0.05$ (one-way analysis of variance). Experiments were performed in triplicate. siRNA, small interfering RNA; shRNA, short hairpin RNA; Fz2, frizzled-2; mock, mock transfected; nega, transfected with negative control; MTS, 3-(4,5-dimethylthiazol-2-yl)-5-(3-carboxymethoxyphenyl)-2-( 4-sulfophenyl)-2H-tetrazolium inner salt.

A

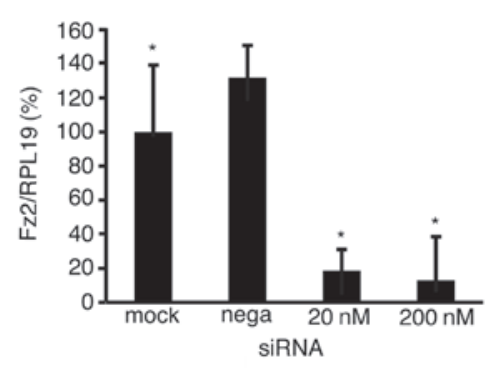

C

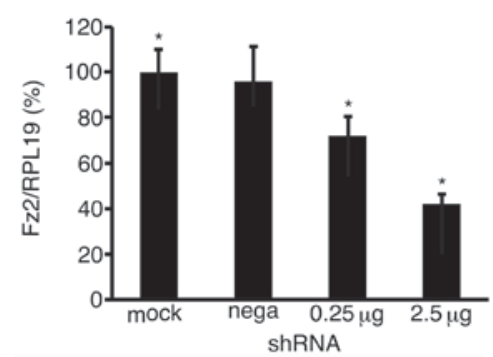

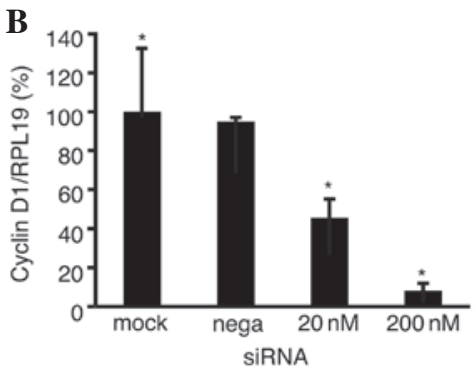

D

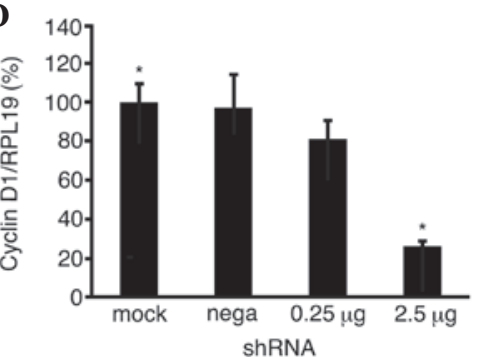

Figure 4. Quantitative PCR. Expression levels of Fz2 and cyclin D1 were analyzed in MIA-Paca2 cells transfected with (A and B) the siRNA or (C and D) shRNA of Fz2. The groups were as follows: Mock, mock transfected; nega, transfected with negative control; $20 \mathrm{nM}$, transfected with $20 \mathrm{nM}$ siRNA; $200 \mathrm{nM}$, transfected with $200 \mathrm{nM}$ siRNA; $0.25 \mu \mathrm{g}$, transfected with $0.25 \mu \mathrm{g}$ shRNA; and $2.5 \mu \mathrm{g}$, transfected with $25 \mu \mathrm{g}$ shRNA. * $<0.05$ (one-way analysis of variance). Experiments were performed in triplicate. PCR, polymerase chain reaction; Fz2, frizzled-2; siRNA, small interfering RNA; shRNA, short hairpin RNA; RPL19, ribosomal protein L19.

The discrepancy between the RT-PCR and quantitative PCR results may be attributed to the saturation of PCR products in the former method. The results of the quantitative PCR were consistent with that of previous studies. In pancreatic cancer patients, Fz2 is upregulated in 8 cases out of 15 (16). In the present study, relative to normal pancreatic tissues, Fz2 was overexpressed in 6 out of the 7 pancreatic cancer cell lines. The results of the quantitative PCR indicate, therefore, that Fz2 is upregulated in pancreatic cancer cell lines more often than in pancreatic cancer tissues. We hypothesize that the upregulation of Fz2 is necessary for cells to proliferate in vitro. Notably, $\beta$-catenin protein levels have been shown to be upregulated in patients whose cancerous pancreatic tissue showed increased expression levels of Fz2 (16), even though the gene encoding $\beta$-catenin was only mutated in $13.3 \%(2 / 15)$ of the patients (16). Using a global genomic analysis, a previous study found that 12 pathways were altered in 24 pancreatic cancer patients, to varying degrees (17). Alteration of the Wnt pathway is not specific to pancreatic cancer, but also occurs in colorectal cancer and hepatocellular carcinoma (18). Fz2 was has been found to be upregulated in $61.5 \%$ (8/13) of pancreatic cancer patients in the absence of the $\beta$-catenin mutation (16). APC is not mutated and $\beta$-catenin is not upregulated in MIA-Paca2 cells, a representative cell line of pancreatic cancer (19). In the present study, cellular transfection with siRNA- and shRNA-Fz2 suppressed the proliferation of the MIA-Paca2 cells, a result that we now expect to obtain in patient pancreatic cancer tissues containing normal APC and $\beta$-catenin.

Cyclin D1 is upregulated in pancreatic cancer tissues compared with surrounding normal tissues (20). Moreover, antisense oligonucleotides specific to cyclin D1 inhibit the growth of pancreatic cancer in vivo (21). Together, these results indicate that pancreatic cancer cell proliferation is suppressed with the inhibition of cyclin D1 expression. In cells of hepatocellular carcinoma, proliferation is suppressed with siRNA-Fz9 and is associated with the decreased expression of cyclin D1 (14). Similarly, in the present study, pancreatic 
cancer cell proliferation and the expression of cyclin D1 were suppressed following siRNA- and shRNA-Fz2 treatment, the latter as revealed by quantitative PCR analysis. The results are, therefore, consistent with previous observations. The main disadvantages of using antisense RNA in cancer treatment are that its half-life is short and its delivery is difficult (22). In contrast to cyclin D1, a cytoplasmic protein, Fz2, is a receptor. A novel molecular therapy for pancreatic cancer may, therefore, be developed using monoclonal antibodies and small molecule receptor inhibitors specific to Fz2. Future studies are likely to analyze the signaling pathway between Fz2 and cyclin D1 in pancreatic cells and tissues.

\section{References}

1. Jemal A, Siegel R, Xu J and Ward E: Cancer statistics, 2010. CA Cancer J Clin 60: 277-300, 2010.

2. Mao C, Domenico DR, Kim K, Hanson DJ and Howard JM: Observations on the developmental patterns and the consequences of pancreatic exocrine adenocarcinoma. Findings of 154 autopsies. Arch Surg 130: 125-134, 1995.

3. Vogel I, Krüger U, Marxsen J, et al: Disseminated tumor cells in pancreatic cancer patients detected by immunocytology: a new prognostic factor. Clin Cancer Res 5: 593-599, 1999.

4. Winter JM, Ting AH, Vilardell F, et al: Absence of E-cadherin expression distinguishes noncohesive from cohesive pancreatic cancer. Clin Cancer Res 14: 412-418, 2008.

5. Iovanna J, Mallmann MC, Gonçalves A, Turrini O and Dagorn JC: Current knowledge on pancreatic cancer. Front Oncol 2: 6, 2012

6. Asuthkar S, Rao JS and Gondi CS: Drugs in preclinical and early-stage clinical development for pancreatic cancer. Expert Opin Investig Drugs 21: 143-152, 2012.

7. Tomizawa M, Shinozaki F, Sugiyama T, Yamamoto S, Sueishi M and Yoshida T: Insulin-like growth factor-I receptor in proliferation and motility of pancreatic cancer. World $\mathrm{J}$ Gastroenterol 16: 1854-1858, 2010.

8. Tomizawa M, Shinozaki F, Sugiyama T, Yamamoto S, Sueishi M and Yoshida T: Insulin-like growth factor I receptor involvement in proliferation of NOR-P1 cells in serum-free media. J Cell Biochem 113: 2714-2720, 2012.
9. Rajpathak SN, Gunter MJ, Wylie-Rosett J, et al: The role of insulin-like growth factor-I and its binding proteins in glucose homeostasis and type 2 diabetes. Diabetes Metab Res Rev 25: 3-12, 2009.

10. Katoh M and Katoh M: WNT signaling pathway and stem cell signaling network. Clin Cancer Res 13: 4042-4045, 2007.

11. Chen HJ, Hsu LS, Shia YT, Lin MW and Lin CM: The $\beta$-catenin/TCF complex as a novel target of resveratrol in the Wnt/ $\beta$-catenin signaling pathway. Biochem Pharmacol 84: 1143-1153, 2012.

12. Wang HY, Liu T and Malbon CC: Structure-function analysis of Frizzleds. Cell Signal 18: 934-941, 2006.

13. Kirikoshi H, Sekihara H and Katoh M: Expression profiles of 10 members of Frizzled gene family in human gastric cancer. Int J Oncol 19: 767-771, 2001.

14. Fujimoto T, Tomizawa M and Yokosuka O: SiRNA of frizzled-9 suppresses proliferation and motility of hepatoma cells. Int $\mathbf{J}$ Oncol 35: 861-866, 2009.

15. Sagara N, Toda G, Hirai M, Terada M and Katoh M: Molecular cloning, differential expression, and chromosomal localization of human frizzled-1, frizzled-2, and frizzled-7. Biochem Biophys Res Commun 252: 117-122, 1998.

16. Zeng G, Germinaro M, Micsenyi A, et al: Aberrant Wnt/beta-catenin signaling in pancreatic adenocarcinoma. Neoplasia 8: 279-289, 2006.

17. Jones S, Zhang X, Parsons DW, et al: Core signaling pathways in human pancreatic cancers revealed by global genomic analyses. Science 321: 1801-1806, 2008.

18. White BD, Chien AJ and Dawson DW: Dysregulation of Wnt $/ \beta$-catenin signaling in gastrointestinal cancers. Gastroenterology 142: 219-232, 2012.

19. Pujal J, Capellá G and Real FX: The Wnt pathway is active in a small subset of pancreas cancer cell lines. Biochim Biophys Acta 1762: 73-79, 2006.

20. Luo Y, Qiu Z, Tian L, et al: Identification of novel predictive markers for the prognosis of pancreatic ductal adenocarcinoma. Hum Pathol 44: 69-76, 2012.

21. Wang JC, Thiere M, Henne-Bruns D, Knippschild U and Kornmann M: Inhibition of pancreatic cancer cell growth in vivo using a tetracycline-inducible cyclin D1 antisense expression system. Pancreas 42: 141-148, 2012.

22. Heidegger I, Pircher A, Klocker H and Massoner P: Targeting the insulin-like growth factor network in cancer therapy. Cancer Biol Ther 11: 701-707, 2011. 